\title{
BM] Global Health Using the Consolidated Framework for Implementation Research to implement and evaluate national surgical planning
}

Saurabh Saluja, ,2,3 Allison Silverstein, ${ }^{2,4}$ Swagoto Mukhopadhyay, ${ }^{2,3,5}$ Yihan Lin,, 2,6 Nakul Raykar, ${ }^{2,7}$ Salmaan Keshavjee, ${ }^{3}$ Lubna Samad, ${ }^{3,8}$ John G Meara ${ }^{2,3,9}$

To cite: Saluja S, Silverstein A, Mukhopadhyay S, et al. Using the Consolidated Framework for Implementation Research to implement and evaluate national surgical planning. BMJ Glob Health 2017;2:e000269. doi:10.1136/ bmjgh-2016-000269

Received 15 December 2016 Revised 22 April 2017 Accepted 24 April 2017

\section{CrossMark}

- Additional material is published online only. To view please visit the journal online (http://dx.doi.org/10.1136/ bmjgh-2016-000269)

For numbered affiliations see end of article.

Correspondence to Dr Saurabh Saluja; sausaluja@ gmail.com

\section{ABSTRACT}

The Lancet Commission on Global Surgery defined six surgical indicators and a framework for a national surgical plan that aimed to incorporate surgical care as a part of global public health. Multiple countries have since begun national surgical planning; each faces unique challenges in doing so. Implementation science can be used to more systematically explain this heterogeneous process, guide implementation efforts and ultimately evaluate progress. We describe our intervention using the Consolidated Framework for Implementation Research. This framework requires identifying characteristics of the intervention, the individuals involved, the inner and outer setting of the intervention, and finally describing implementation processes. By hosting a consultative symposium with clinicians and policy makers from around the world, we are able to specify key aspects of each element of this framework. We define our intervention as the incorporation of surgical care into public health planning, identify local champions as the key individuals involved, and describe elements of the inner and outer settings. Ultimately we describe top-down and bottom-up models that are distinct implementation processes. With the Consolidated Framework for Implementation Research, we are able to identify specific strategic models that can be used by implementers in various settings. While the integration of surgical care into public health throughout the world may seem like an insurmountable challenge, this work adds to a growing effort that seeks to find a way forward.

\section{INTRODUCTION}

Surgical care remains out of reach for five billion people - the majority of whom live in low-income and middle-income countries (LMICs). To confront this inequity, the Lancet Commission on Global Surgery (LCoGS) published Global Surgery 2030 in April 2015. This report defined a vision for global surgery and outlined a list of six surgical indicators (table 1) with corresponding targets to assess the status of an individual country's surgical system. In addition, the report proposed the development of national surgical plans (NSPs) as a means to incorporate surgical care into a country's health agenda. For these

\section{Key questions}

What is already known about this topic?

- There exists an emerging movement in global health that seeks to incorporate surgical care into public health planning.

- Prior studies have investigated factors that shape the priority for global surgical care, as well as looked at how to effectively execute organisation change.

What are the new findings?

- We build on existing work by evaluating our intervention via the Consolidated Framework for Implementation Research tool.

- We describe various factors that influence adoption of global surgery and suggest implementation models for integration of surgical care in diverse settings.

Recommendations for policy

- As surgery becomes a global public health priority, a clear framework and models designed to aid in the implementation of this intervention are critical to success

- Our work will help leaders worldwide guide their community or country to overcome distinct challenges and move forward in integrating surgery into public health planning.

NSPs, the report provided a framework for surgical system development that included recommendations for improvement, monitoring and evaluation (available at http:// www.lancetglobalsurgery.org/implementation-tools). This framework has served as a basis for national surgical strategic planning that is currently underway in a number of countries, including India, Madagascar, South Africa, Zambia and several island-nations in the Western Pacific Islands. As the importance of surgical care in global public health gains traction in more places, it will be important for groups that are initiating these policy discussions to have a rigorous process for developing their approach and evaluating 
Table 1 Six Lancet indicators for measurement and assessment of global surgical systems

Group 1: Preparedness for surgical and anaesthesia care

$\begin{array}{ll}\text { Access to timely essential surgery } & \begin{array}{l}\text { Proportion of the population that can access, within } 2 \text { hours, a facility that } \\ \text { can do caesarean delivery, laparotomy and treatment of open fracture (the } \\ \text { Bellwether procedures) }\end{array} \\ \text { Specialist surgical workforce density } & \begin{array}{l}\text { Number of specialist surgical, anaesthetic and obstetric physicians who } \\ \text { are working per } 100000 \text { population }\end{array}\end{array}$

Group 2: Delivery of surgical and anaesthesia care

Surgical volume

Procedures done in an operating theatre, per 100000 population per year

Perioperative mortality rate

All-cause death rate before discharge in patients who have had a procedure in an operating theatre, divided by the total number of procedures, presented as a percentage

Group 3: Impact of surgical and anaesthesia care

Protection against impoverishing expenditure

Proportion of households protected against impoverishment from direct out-of-pocket payments for surgical and anaesthesia care

Protection against catastrophic expenditure

Proportion of households protected against catastrophic expenditure from direct out-of-pocket payments for surgical and anaesthesia care

Adapted from the Lancet Commission on Global Surgery.

their progress. The field of implementation science provides the academic space for this discourse.

\section{IMPLEMENTATION SCIENCES IN GLOBAL SURGERY}

Implementation science is a field that studies the methods by which research, evidence-based practices and interventions are incorporated into healthcare improvement. ${ }^{2}$ These methods can be characterised along a spectrum of complexity (eg, discrete vs multifaceted), can have varied actors and targets, and can occur at differing organisational levels. ${ }^{34}$ Our intervention, put succinctly, is to incorporate surgical care into broader public health planning. This multifaceted intervention is not set within a clinic or hospital, but instead occurs in a health system - be it at the national level, the district level or within a network of private hospitals. Further, the intervention we seek to characterise has a broad array of implementers that include, but are not limited to, academic research consortia, individual clinicians or Ministries of Health.

Despite the complexity of the intervention - or perhaps because of it - we believe the implementation science field offers a robust set of tools that are useful in understanding this heterogeneous process. The Consolidated
Framework for Implementation Research (CFIR) is one such implementation science tool that can facilitate these efforts. ${ }^{5}$ Originally described by Damschroder in 2009, the CFIR serves as 'an overarching typology - a list of constructs to promote theory development and verification about what works where and why across multiple contexts'. In the CFIR, five domains are described to interact and influence implementation effectiveness: the intervention, individuals, the inner setting, the outer setting and the implementation process (table 2). By applying this framework to the global surgery movement, we seek to develop a richer understanding of the factors that impede or support the incorporation of surgery into public health and develop models for doing so in a diversity of settings.

\section{A CONSULTATIVE SYMPOSIUM}

This analysis is based on a consultative symposium in which experts were invited to share their experiences with national surgical planning in a variety of countries and discuss key factors for implementation. The symposium, entitled Global Surgery: Towards Equitable Surgical Systems, was hosted at the Harvard Medical School Center

Table 2 Definitions of the domains of the Consolidated Framework for Implementation Research

\begin{tabular}{|c|c|}
\hline Domain & Definition \\
\hline $\begin{array}{l}\text { Characteristics of the } \\
\text { intervention }\end{array}$ & $\begin{array}{l}\text { The 'core components' - that is, the essential elements of the intervention - and the 'adaptable } \\
\text { periphery' - that is, the adaptable elements in which the intervention occurs }\end{array}$ \\
\hline Individuals & $\begin{array}{l}\text { The individuals responsible for carrying out the intervention or otherwise related to the } \\
\text { intervention, their agency, and their relationships to each other and the intervention }\end{array}$ \\
\hline Inner setting & $\begin{array}{l}\text { The 'structural, political, and cultural context through which the intervention proceeds' and the } \\
\text { relationship between these elements }\end{array}$ \\
\hline Outer setting & The 'economic, political, and social context within which an organization resides' \\
\hline Implementation process & The active process through which the desired changes are achieved \\
\hline
\end{tabular}


for Global Health Delivery - Dubai in March 2016 and had the expressed purpose of "take[ing] national surgical planning from an academic exercise to an actionable process'. Participants were informed that the goal was to 'develop a roadmap by which current and new partners can embark on national surgical planning processes in their home countries'.

Participants at the symposium were selected to include those with prior international involvement with global surgery efforts, those representing LMICs, those representing countries with ongoing national surgical planning efforts and those representing various sectors of care delivery (eg, public sector, private sector, non-governmental organisations and industry). The symposium was structured around the five previously mentioned components of the NSP: infrastructure, workforce, service delivery, information management and financing. Each of these was presented by a content expert and then discussed in small-group sections. Within each of the sessions, participants noted challenges unique to their setting as well as shared challenges; similarly, they identified solutions that might be particular to a given country but also solutions that could cross borders. These informed a discussion geared towards the development of models for how national surgical planning can be initiated in a variety of settings around the world. Importantly, there was consensus around the importance of collecting six previously identified indicators of surgical system strength as they allow for benchmarking and evaluation of progress of NSP initiatives (table 1).

A detailed description of the symposium discussion is available in the symposium proceedings. ${ }^{6}$ The participants represented the experience of numerous countries engaged or planning to engage in national surgical planning. Thus the discussions represent a post-hoc analysis of implementation efforts in multiple settings as well as discussions about how to maximise future implementation. These proceedings, which were recorded by a rapporteur and developed in collaboration with the authors of this manuscript, were annotated by the lead author to determine which of the CFIR constructs were relevant to the conference proceedings. Because the CFIR was not an a priori framework for the symposium, not all constructs were explicitly discussed and therefore not all constructs are presented here.

\section{UNDERSTANDING NATIONAL SURGICAL PLANNING USING THE CFIR}

The use of the CFIR allows stakeholders to anticipate barriers and facilitators (ie, determinants) to implementation so they can strategically plan for key challenges that occur in integrating surgical care into public health planning. First, the CFIR requires clearly defining the characteristics of the intervention in terms of its core components and an adaptable periphery. Many authors have previously noted that implementation research lacks a unified language and clear definition of the components of an intervention. ${ }^{4}$ While we had defined our intervention at the outset as the incorporation of surgical systems into public health planning, we were able to further clarify what this meant by building consensus around two core components: the use of six surgical indicators and the use of the LCoGS framework for an NSP. While these are not yet empirically linked to the improvement of surgical systems, they were the key policy outputs from the LCoGS and are believed to be essential for measurement and planning. The former incorporates existing evidence and expert opinion on surgical systems to identify key indicators and associated benchmarks. The latter is not an exhaustive or complete list for national surgical planning, but rather a framework that is meant to be adapted for use in engaging in national surgical planning.

Moreover, we identified aspects of an adaptable periphery that are critical to making these processes locally relevant. Participants noted that where data are more readily available, this adaptable periphery could result in an expansion of the six indicators to include disaggregation, more detailed information (eg, rate of surgical site infection) and procedure-specific data (eg, rate of fistula in cleft repair). They additionally noted different ways in which data could be collected. Examples of these include national databases, ${ }^{7}$ field research, ${ }^{8}$ insurance databases and trauma registries. ${ }^{9}$ Additionally, participants voiced beliefs that, in some countries, planning on the scale of states, cities or hospital systems may be more relevant and practical than national surgical planning. Similarly, while the indicators are meant to be collected at the national level, a number of participants noted that using insurance databases, information from professional societies or state-level data may actually prove more practical. Thus, while we have used the terminology 'National Surgical Plans', we recognise the level of engagement is part of the adaptable periphery, and that in many cases subnational implementation will serve as a first step to the incorporation of surgical care into public health planning.

The CFIR notes the importance of individuals in implementing an intervention, specifically noting four types of implementation leaders: opinion leaders, internal implementation leaders, champions and external change agents. ${ }^{5}$ We focus on the role of a 'local champion' who believes strongly in the cause, will align himself or herself with the intervention and understands local ecosystems. This person, who may or may not be formally integrated into a governing apparatus, is a critical change agent. He or she may be a clinician, a patient advocate, a policy maker or anyone else who believes strongly that surgical care should become part of public health planning and is positioned to make changes in that direction.

The inner setting includes institutions, networks and the climate that directly interacts with the intervention. In our case these include the groups that provide, pay for and receive surgical care, the bodies that govern health systems, and the relationships between each of these. There are numerous possible institutions and formal or informal organisations that may comprise the inner 


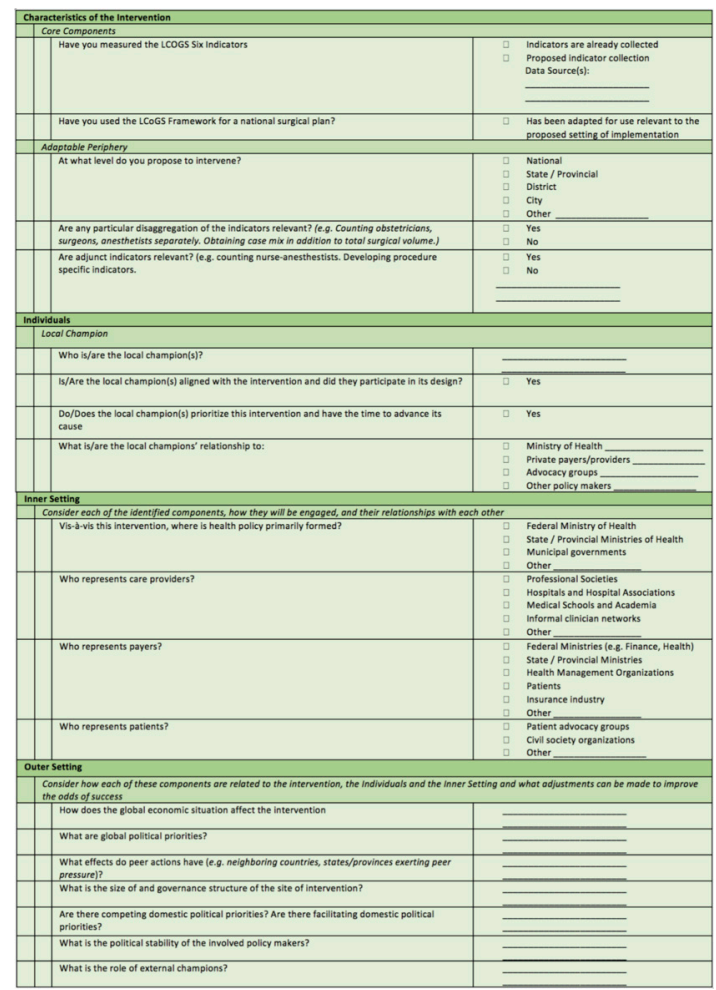

Figure 1 A worksheet for understanding national surgical planning using the Consolidated Framework for Implementation Research. LCoGS, Lancet Commission on Global Surgery.

setting and thus should be explicitly included in national surgical planning.

The outer setting is defined as the 'economic, political and social context within which an organization resides'. These may be international or domestic. In the international sphere, the global economy is particularly important due to the effect it has on currency fluctuations that in turn constrain infrastructure development and equipment purchasing. Global political priority and peer actions from neighbouring countries can also have the effect of stimulating governments to engage in national surgical planning. For example, the passage of World Health Assembly Resolution 68.15, 'strengthening emergency and essential surgical care as a part of universal health coverage', may increase pressure on countries to engage in national surgical planning. ${ }^{10}$ Domestically, the size and governance structure of a country are critical to deciding at what level implementation would occur. Similar to global political priority, domestic political priority is also a key consideration: are other more pressing health issues like an infection outbreak likely to limit interest in national surgical planning?

Collectively, the characteristics of the intervention, the individuals, the inner setting and the outer setting act as parameters - although not entirely immovable ones
- within which an implementer must act. The subsequent action, in turn, is the implementation process that the implementer selects to move forward strategically. The worksheet proposed in figure 1 allows for systematic consideration of these parameters prior to choosing an implementation process. The worksheet is not exhaustive nor is it designed to be prescriptive, but rather it is meant to help implementers identify critical aspects of national surgical planning, which can then inform strategic decision making about the implementation process.

\section{Strategic choice of implementation process: models for action}

We evaluated the real-world implementation processes that were used for NSP efforts in Zambia, Cabo Verde, South Africa, India and Madagascar. Each of these processes took on slightly different forms based on the domains of the CFIR discussed above. While each process had its own nuances and variables, there were two broad models that emerged: a top-down approach (figure 2) and a bottom-up approach (figure 3). In the top-down approach, the Ministry of Health drives implementation, whereas in the bottom-up approach implementation is driven by civil societies or professional organisations. While the CFIR is primarily a determinant framework, we describe the implementation process as a strategic choice that can follow from the other more determinant domains. Each model describes a different process that has been used by previous implementers and, in turn, may be strategically chosen for future implementation in a different locale.

\section{The top-down model}

In the top-down model, early engagement with the Ministry of Health is a crucial element. External champions may reach out to local champions and help generate energy, excitement and dedication to the effort. This local champion may sit within or outside of the Ministry of Health. From here, stakeholders are identified and technical working groups are formed, allowing for consideration of existing data on surgical systems as well as integrating the lived experiences of stakeholders into the framework for the NSP. As stakeholders are continually broadened, a national surgical forum is planned. This forum has core outputs, which include new partnerships, further data collection and a policy document.

Within this broad structure, there may be many variations as to how specifically an implementer would proceed. For example, the role of the local champion, the timeframe between initial discussions and a national surgical forum, the input and collection of data, and the timing of development of a strategic document may all vary. While not meant to be prescriptive, this model suggests one approach that can work well when there is a high level of ministry engagement and the public sector is dominant. 


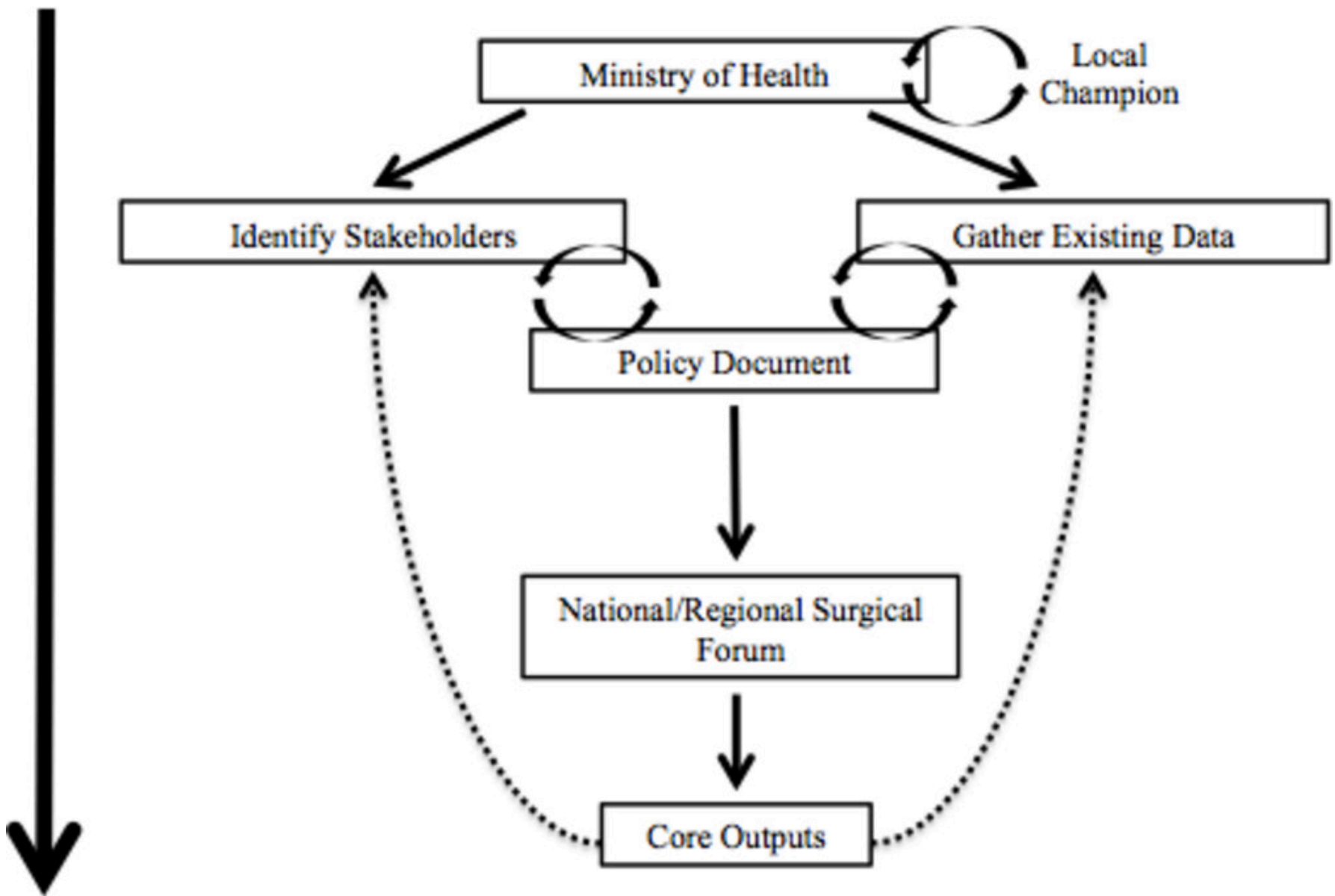

Figure 2 Top-down approach to national surgical planning.

The bottom-up model

The bottom-up model is characterised by early engagement with a professional body. The local champion may not be connected to the Ministry of Health but instead have ties to professional organisations, research institutions or patient advocacy groups. Here, iterative consultations are held at the 'grassroots' provider level. Eventually, a policy document is developed and the stakeholder group is broadened; the goal remains hosting a national surgical forum that includes a broad array of participants, such as the Ministry of Health or major private payers. While numerous elements of the bottom-up process are variable, both India and South Africa's processes involved the early elaboration of a policy document. These documents serve as a tangible product and allow grassroots efforts to further broaden stakeholder engagement. They may take the form of terms of references, consensus statements or strategic plans. This bottom-up model appears to be well-suited for larger countries in which delivery of care is highly decentralised.

While both these models are centred around core concepts (the six surgical indicators and the LCoGS framework for surgical planning), their specific outputs may take multiple forms. They may generate or strengthen research groups to focus on surgical data collection, they may foster partnerships or pilot projects for surgical infrastructure expansion, or they may result in the writing of an official NSP document and shared vision for the future of the country's surgical system. Nevertheless, the models can guide a broad array of implementers to initiate important policy-level discussions in their country. Moreover, these models respond to a key challenge identified in implementation science literature: the need to define and analyse implementation strategies and justify their selection. ${ }^{411}$

As an example that illustrates the utility of these models, we consider a hypothetical implementer looking to champion surgical care as a part of public health in Brazil (case 1, online supplementary appendix 1) and the real-life example of Zambia (case 2, online supplementary appendix 2). In these examples, we see how the four determinant domains of the CFIR (characteristics, individuals, inner and outer settings) can lead implementers to a rational choice of implementation process.

\section{LIMITATIONS OF CFIR AND COMPLEMENTARY FRAMEWORKS}

As demonstrated in the examples above, the CFIR provides a useful approach to strategically engage in national surgical planning. Nevertheless, the CFIR has its limitations. Damschroder et a $\bar{l}$ acknowledge that in 


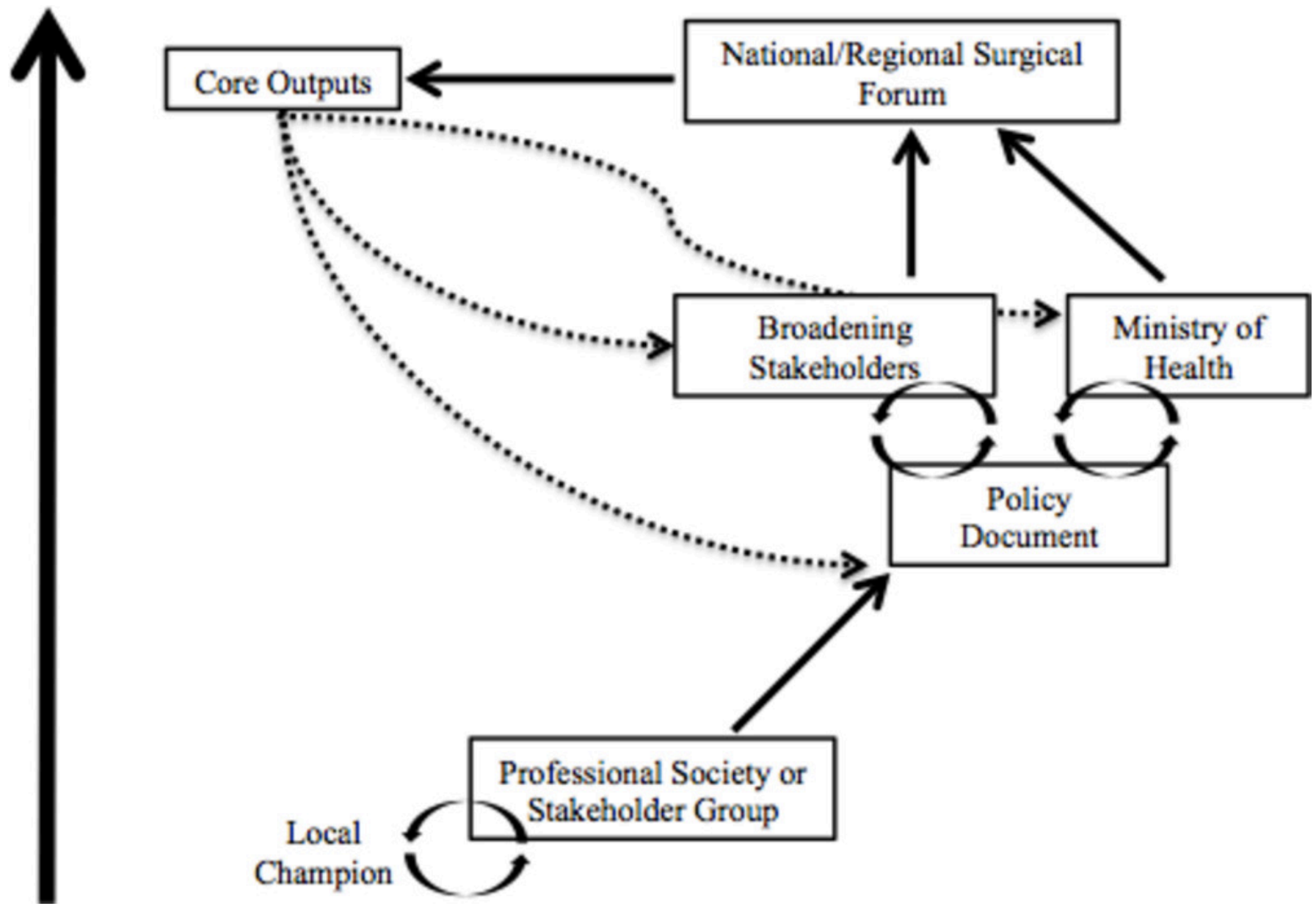

Figure 3 Bottom-up approach to national surgical planning.

implementation science literature, 'little research has been done to gain understanding of the dynamic interplay between individuals and the organisations [they seek to change]'. Implementers may choose to draw on literature from business administration to fill this gap. Kotter ${ }^{12}$ for example, identifies eight stages for leading organisational change: (1) establishing a sense of urgency, (2) forming a guiding coalition, (3) creating a vision, (4) communicating the vision, (5) empowering others to act, (6) planning and creating short-terms wins, (7) consolidating improvements and (8) institutionalising new approaches. A local champion could draw on a shared international vision (eg, 'Universal access to safe, affordable surgical and anesthesia care when needed' as articulated by the LCoGS) and use these stages to adapt and advance that vision in their local setting.

Shawar and Shiffman have described a policy framework to identify global political priorities for global health initiatives, including for global surgery. ${ }^{13}{ }^{14}$ In their framework, Shawar and Shiffman describe 'actor power', 'ideas', 'political contexts' and 'issue characteristics' as key elements that shape support for a global health initiative. Conceptually, the elements of political priority align with aspects of the CFIR and may provide an analytic approach to a deeper view of the inner setting and outer setting. Shawar et al ${ }^{13}$ determine that 'global surgery faces several challenges' due to being a fragmented community, having a paucity of surgical data and not having an issue aligned with funding schemes. While the political priority framework identifies key challenges or constraints in the field of global surgery, the CFIR builds on their work by suggesting specific implementation models that respond to political realities.

\section{FUTURE DIRECTIONS}

While our analysis provides a systematic approach to understanding and leading surgical planning efforts, it is meant to be augmented by future research in implementation sciences.

Importantly, our implementation processes (ie, top-down and bottom-up models) have not yet been tested. Proctor et $a l^{?}$ note that an "unresolved issue in the field of implementation science is how to conceptualize and evaluate success'. They distinguish between service and client outcomes, which are distal, and implementation outcomes, which are the necessary intermediate steps and are revealed at multiple stages along the process of achieving the targeted changes. In our case, the service 
Case 1: Contextualising the Consolidated Framework for Implementation Research in Brazil

Brazil is South America's largest country with nearly 180 million people. The country has a large, publicly funded health system, the Sistema Unico de Saude (SUS), that coexists with a large number of private providers; while all citizens have public insurance, between $20 \%$ and $25 \%$ of the population also has private insurance. ${ }^{17}$ In considering the outer setting in Brazil, an implementer may note that there is an ongoing political and economic crisis that is exemplified by a devalued currency and the recent impeachment of former President Dilma Rousseff. ${ }^{18}$ Further, the recent scourge of Zika virus and the Olympics may have directed public health attention to the prevention of infectious diseases. Collectively, the outer setting in Brazil suggests an environment that - at least at the national level — may not be amenable towards ambitious or expensive new endeavours.

Within the inner setting, however, the implementer may note that SUS is the main provider of care for nearly three-fourths of the population; that while SUS is financed by the federal government, it is administered at the state and municipal level; and that physicians have strong organisational societies. Further, Brazilian public services promote transparency and much of the data collected by SUS are publicly available. These factors may suggest that collecting data on surgical indicators as reported by SUS is possible. Further, an implementer could seek out local champions at the state or municipal level where policies surrounding administration of SUS are formed.

The implementer may conclude that, of the models developed, a bottom-up approach may prove more effective; they may choose to target their action towards a state government instead of the national one, and they may engage a professional society to identify how surgical care can inform the public health discourse.

and client outcomes would include, for example, reduction in mortality from surgical disease and improved equity of surgical access, respectively. These outcomes, however, may take years to realise. Thus, it is important to identify intermediate metrics to gauge success. These may include, for example, measuring whether developing data platforms were successful or whether policy makers adopted core messages.

Proctor et al created a taxonomy to assess implementation outcomes in which they suggest the stages during which measurement of the outcome is most relevant. They identify critical stages of measurement (ie, acceptability, adoption, appropriateness, feasibility, fidelity, implementation cost, penetration and sustainability) which, in global surgery, could be adopted to create benchmarks to measure implementation outcomes. ${ }^{3}$

Further, other research groups have developed alternative qualitative and quantitative CFIR measures. For example, Gustafson $e t a l^{15}$ developed a model that used survey data to predict the potential for successful implementation of a health system change and to identify obstacles or favourable factors for such implementation. This type of model could help implementers identify potential obstacles early on and aid their chances of meeting their goals. Aarons $e t a l^{16}$ looked at how leadership characteristics influenced effective implementation of practices. For our proposed models, local champions
Case 2: Contextualising the Consolidated Framework for Implementation Research in Zambia

Zambia is a southern African country with a population of 14.5 million people. Throughout a series of National Health Strategic Plans, health has been prioritised as a major area for social investment and economic growth, and forms a critical step towards the national goal of attaining a higher human development status by 2030. At the 68th World Health Assembly (WHA), Zambia proposed a resolution for universal access to emergency and essential surgical care. ${ }^{10}$ WHA Resolution 68.15 was supported by 197 member states. Since then, Zambia has embarked on its own national surgical plan. Surgical care in Zambia is primarily delivered through a public system that exists in a tiered structure, with 12 third-level hospitals serving as referral centres. The University Teaching Hospital in Lusaka is the principal site for medical education and serves as the highest level referral centre. Additionally, in Zambia, non-governmental organisations have an important role in healthcare and are tasked with augmenting services in different regions; these 'cooperating partners' join together on a regular basis to discuss national health issues and coordinate healthcare delivery. There is a substantial amount of data collected from the various hospitals, but much of it is fragmented and is only recently being reported into a nascent central repository.

An implementer in Zambia would likely choose the top-down model, knowing that this is a relatively small country with most of the care managed from Lusaka. They would see the outer setting as quite favourable to national surgical planning given the high political value given to essential surgical care in Zambia. The presence of a National Health Strategic Plan also provides opportunity for development of a formal, institutionalised national surgical plan that is adapted to Zambian circumstances. The inner setting, which includes a very involved Ministry of Health, also points to some key stakeholders who should be consider early on, notably the 'cooperating partners'. Further, it suggests that the intervention may choose to focus initial efforts on strengthening ongoing indicator collection activities.

are critical to the successful implementation of global surgery; as such, consideration of how effective of a leader these individuals may be is also critical. Such evaluation can help provide information about what leads to the success or failure of implementation efforts. Further work in global surgery should adapt these existing tools to develop measurable implementation outcomes that allow for benchmarking progress and planning future strategies as national surgical planning efforts are underway.

\section{CONCLUSION}

We use the CFIR to understand an emerging movement in global health that seeks to incorporate surgical care into public health planning. In doing so, we clearly define our intervention, gain an understanding of the external and internal factors that impede or facilitate adoption of the intervention, and describe implementation processes. In our analysis, the implementation process is not purely deterministic; we describe two models of implementation process that can be strategically selected based on the other four domains.

Integration of surgical care into public health priorities and actualisation of this effort can seem like a daunting 
challenge. However, our analysis helps to simplify a rather complicated intervention by highlighting important factors to consider and by creating models for a stepwise course of action. Moreover, we believe our analysis can be used in myriad settings with differing surgical systems, public health priorities and support systems. While the integration of surgery into public health planning continues to face distinct challenges, our analysis adds to a growing academic literature that identifies a way forward.

In the top-down model, a local champion either within or affiliated with the Ministry of Health engages stakeholders and works to gather existing data to eventually elaborate a policy document. This document is considered in a national forum. The core outputs of this document may include mechanisms to collect more data, bringing in of new stakeholder or funders, or development of formal surgical plans that are incorporated into legislation. Importantly, these outputs feed back on a process that is ultimately iterative.

In the bottom-up model, a local champion leverages domestic societal groups such as professional organisations to discuss the importance of surgical planning. Through an internal consultation, a policy document is often elaborated allowing for the group to broaden stakeholders and bring new contributors to the document. After an iterative review with new stakeholders, the document is brought to a national level where the Ministry of Health and other drivers of health policy are integrated into the process. The core outputs of this document may include mechanisms to collect more data and bring in new stakeholder or funders. Similar to the top-down approach, outputs feed back information to be incorporated in future policy documents.

\section{Author affiliations \\ ${ }^{1}$ Department of Surgery, Weill Cornell Medicine, New York, USA \\ ${ }^{2}$ Department of Global Health and Social Medicine, Program in Global Surgery and Social Change, Harvard Medical School, Boston, USA \\ ${ }^{3}$ Center for Global Health Delivery - Dubai, Harvard Medical School, Dubai, UAE \\ ${ }^{4}$ Department of Medical Education, University of Miami, Miami, USA \\ ${ }^{5}$ Department of Surgery, University of Connecticut, Hartford, USA \\ ${ }^{6}$ Department of Surgery, University of Colorado, Denver, USA \\ ${ }^{7}$ Department of Surgery, Beth Israel Deaconess Medical Center, Boston, USA \\ ${ }^{8}$ Department of Surgery, Indus Hospital, Karachi, Pakistan \\ ${ }^{9}$ Department of Plastic Surgery, Boston Children's Hospital, Boston, USA}

Twitter @ssalujaMD @agsils @Swaggermuk @yihanlinMD @NakulRaykar @ JohnMeara

Acknowledgements The authors would like to acknowledge Anna Nicholson for development of the proceedings.

Contributors SS, AS, LS and JGM contributed to the design, analysis, interpretation and writing of this manuscript. SM, YL, SK and NR contributed to the analysis and interpretation of the work presented in this manuscript. All authors participated in the writing or revision of the manuscript and agree to be accountable to the accuracy and integrity of the work presented.

Funding The described symposium was funded by the Harvard Medical School Center for Global Health Delivery - Dubai. There were no additional funding sources for this work.

\section{Competing interests None declared.}

Provenance and peer review Not commissioned; externally peer reviewed.

Open Access This is an Open Access article distributed in accordance with the Creative Commons Attribution Non Commercial (CC BY-NC 4.0) license, which permits others to distribute, remix, adapt, build upon this work non-commercially, and license their derivative works on different terms, provided the original work is properly cited and the use is non-commercial. See: http://creativecommons.org/ licenses/by-nc/4.0/

(c) Article author(s) (or their employer(s) unless otherwise stated in the text of the article) 2017. All rights reserved. No commercial use is permitted unless otherwise expressly granted.

\section{REFERENCES}

1. Meara JG, Leather AJM, Hagander L, et al. Global surgery 2030: evidence and solutions for achieving health, welfare, and economic development. Lancet 2015;286:569-624.

2. Eccles MP, Mittman BS. Welcome to Implementation Science. Implementation Science 2006;1:1:1.

3. Proctor E, Silmere H, Raghavan R, et al. Outcomes for implementation research: conceptual distinctions, measurement challenges, and research agenda. Adm Policy Ment Health 2011;38:65-76.

4. Proctor EK, Powell BJ, McMillen JC. Implementation strategies: recommendations for specifying and reporting. Implement Sci 2013;8:139.

5. Damschroder LJ, Aron DC, Keith RE, et al. Fostering implementation of health services research findings into practice: a consolidated framework for advancing implementation science. Implement Sci 2009;4:50.

6. Saluja S, Makuhopadhyay RNP, et a/Global surgery towards equitable surgical systems: Harvard Center for Global Health Delivery Dubai 2016. Available at http://ghd-dubai.hms.harvard. edu/files/ghd dubai/files/globalsurgery towardsequitablesurg icalsystems.pdf (Accessed Jan 11, 2017).

7. Massenburg B, Saluja S, Jenny $\mathrm{H}$, et al. Assessing the brazilian surgical system with six surgical indicators. BMJ Global Health. In Press.

8. Bruno $\mathrm{E}$, White $\mathrm{M}$, Baxter $\mathrm{L}$, et al. An evaluation of preparedness, delivery and impact of surgical and anaesthesia care in Madagascar: a framework for a national surgical plan. World J Surg 2016.

9. Peck G, Saluja S, Blitzer D, et al. Using global surgical indicators to improve trauma care in Latin America. Bulletin American College of Surgeons 2017;102:11-17.

10. Strengthening emergency and essential surgical care and anaesthesia as a component of universal health coverage. World Health Assembly 2015;68.15.

11. Nilsen P. Making sense of implementation theories, models and frameworks. Implement Sci 2015;10:53.

12. Kotter JP. Leading Change: why transformation efforts fail. Harv Bus Rev 1995:57-67.

13. Shawar YR, Shiffman J, Spiegel DA. Generation of political priority for global surgery: a qualitative policy analysis. Lancet Glob Health 2015;3:e487-e495.

14. Shiffman J, Smith S. Generation of political priority for global health initiatives: a framework and case study of maternal mortality. Lancet 2007:370:1370-9.

15. Gustafson DH, Sainfort F, Eichler M, et al. Developing and testing a model to predict outcomes of organizational change. Health Serv Res 2003;38:751-76.

16. Aarons GA, Ehrhart MG, Farahnak LR. The Implementation Leadership Scale (ILS): development of a brief measure of unit level implementation leadership. Implement Sci 2014;9:45.

17. suplementar Dados do setor de saúde. 2015. Accessed Nov 13, 2015. Available at: http://www.ans.gov.br/aans/noticias-ans/ numeros-dosetor/3030-dados-do-setor-de-saude-suplementar.

18. Romero S. Dilma Rousseff is ousted as Brazil's president in impeachment vote, 2016. Available at. http://www.nytimes.com/ 2016/09/01/world/americas/brazil-dilma-rousseff-impeachedremoved-president.html. (Accessed Nov 13, 2015). 